\title{
Gherkin Downy Mildew Disease and Its Response to Different Fungicides
}

\author{
Sanjay Bairagi ${ }^{1 *}$, P.S. Nath ${ }^{2}$, M.K. Pandit ${ }^{3}$ and Arindam Das ${ }^{4}$ \\ ${ }^{1}$ College of Agriculture, Extended Campus of Bidhan Chandra Krishi Viswavidyalaya, \\ Chhatna-722132, West Bengal, India \\ ${ }^{2}$ Department of Plant Pathology, ${ }^{3}$ Department of Vegetable Crops, Bidhan Chandra Krishi \\ Viswavidyalaya, Mohanpur-741252, West Bengal, India \\ ${ }^{4}$ Division of Vegetable Crops, ICAR-Indian Institute of Horticultural Research, Bengaluru- \\ 560089, Karnataka, India \\ *Corresponding author
}

\begin{tabular}{|l|}
\hline Ke y w or d s \\
Gherkin, Downy \\
mildew, Fungicide, \\
Control and Yield \\
\hline Article Info \\
\hline $\begin{array}{l}\text { Accepted: } \\
\text { 10 February } 2019 \\
\text { Available Online: } \\
\text { 10 March } 2019\end{array}$ \\
\hline
\end{tabular}

\section{A B S T R A C T}

Gherkin (Cucumis sativus var. anguria) is a vine type cucumber, which is a cucurbit. Gherkin differs from cucumber by its morphology. Downy Mildew is a devastating disease of gherkin, caused by the pathogen Pseudoperonospora cubensis and the pathogen primarily infects leaves, decreased in photosynthesis resulting both yield and quality of fruits reduced. The present investigation was done to control the downy mildew disease of gherkin and to check the loss of gherkin fruit yield and it was carried out with the $F_{1}$ hybrid Ajax in plots of $5 \mathrm{~m} \times 4 \mathrm{~m}$ size accommodating forty plants/plot in Randomized Block Design with three replications with seven treatments during rabi season of 2018 . The appearance of the first visible symptom of the disease of Gherkin was recorded at 37 Days after sowing (DAS). The fungicide Equation Pro $42 \mathrm{SC}$ (262.50 g a.i./ha) showed significant minimum disease severity i.e. $11.40 \%$ and maximum disease control i.e $82.41 \%$ over control, followed by $\mathrm{T}_{3}$ (210 $\mathrm{g}$ a.i./ha) where, disease severity is $14.25 \%$ and disease control is $76.24 \%$ over $\mathrm{T}_{9}$ (control plot). $\mathrm{T}_{4}$ and $\mathrm{T}_{3}$ are at par. The significant lowest disease incidence and highest control was recorded in $\mathrm{T}_{4}$ (Equation Pro 42 SC@262.5 g a.i/ha)and $\mathrm{T}_{3}$ (Equation Pro $42 \mathrm{SC} @ 210.0 \mathrm{~g}$ a.i/ha) treated plots. After 70 days of sowing 13.33 percent disease incident was observed in $\mathrm{T}_{4}$ treated plants and it is same in $\mathrm{T}_{3}$.Curzate M8 72 and Ridomyl $72 \%$ also showed significant disease control percentage i.e $74.01 \%$ and $70.74 \%$, respectively. Significant higher yield was found when Equation Pro $42 \mathrm{SC}$ (262.50 g a.i. /ha) sprayed over crop and is at par with $\mathrm{T}_{3}$ treated plots. Equation Pro 42 SC showed no phytotoxic symptom sprayed to the gherkin crop up to 4 numbers of spraying at their different dosages.

\section{Introduction}

Gherkin (Cucumis sativus var. anguria) is a vine type cucumber, which is a cucurbit.
Gherkin differs from cucumber by its morphology. Fruits of gherkin are small in size compare to cucumber and darker in colour. Length of fruits varies from $4.0 \mathrm{~cm}$ to 
$6.0 \mathrm{~cm}, 1.5-2.0 \mathrm{~cm}$ diameter and dark green in colour. Prominent morphological difference is that, it has rough or undulated surface on fruits, while comparatively smooth fruit surface in cucumber. It is matures 50-60 days after sowing, average yield 10-12 t/ha and due to infestation of downy mildew disease there is reduction of yield and if it is devastating the total yield may be loss. To control fruit loss different fungicides can be used. Ajax is a F1 hybrid very uniform with soft spines that rub off easily, Fruits are olive-green in colour and will attain large size if not harvested at proper stage, making them unsuitable for marketing, Ajax produces predominantly female flowers so has a very high yield potential. The There is a growing worldwide demand for pickled gherkins, brine fermentation is becoming popular, because it is an important preservation method due to its sensory qualities, processing strategies and economic considerations. Texture is one of the most important quality attributes of pickling cucumbers which influences on product acceptance and quality.

Downy Mildew is a devastating disease of gherkin, caused by the pathogen Pseudoperonospora cubensis and the pathogen primarily infects leaves, decreased photosynthesis in gherkin plant resulting both yield and quality of fruits reduced. Due to severe infestation defoliation of leaves may lead, growth of plants stunted, even entire plant may be killed (Bernherdt et al., 1988).

Apart from the leaf wetness essential for infection, the factors determining disease progress are rate of foliage growth and physiological age of the host, amount of primary inoculum available, light, and the rate at which lesions necrotize (Palti and Cohen, 1980). The quantum of crop loss depends on the stage of crop growth at which attack takes place. General symptoms are yellow, water soaked lesions or spots or angular patches on upper surface of leaf and on the corresponding lower surface, grayish or purplish downy growth is observed. Hausbeck et al., also coined that yellow water soaked lesion seen on the upper surface of leaf if it is affected by downy mildew disease. Later grayish or purplish colour turns to black because of more sporulation. Pathogen producing zoospores which are biflagellate, source of infection spread by wind, rain splash, farm equipments, animals, human etc. and maximum disease observed at cool humid climate, foggy weather, prevailing wet condition. Keinath et al., also reported that humidity is one of the limiting factor for cucumber or cucurbits production. At high atmospheric temperature condition development of downy mildew disease slow down, even restricted (Hansen 2000).

\section{Materials and Methods}

The present investigation was carried out at the Instructional Farm, Jaguli, Bidhan Chandra Krishi Viswavidyalaya, with the $F_{1}$ hybrid Ajax in plots of5m $\mathrm{x} 4 \mathrm{~m}$ size accommodating fourty plants/plot, spacing given was $100 \mathrm{~cm} \times 50 \mathrm{~cm}$, in Randomized Block Design with three replications with seven treatments during rabi season of 2018.

Date of sowing was 05.10.2018. Four sprays were done at 40, 50, 60 and 70 days after sowing by means of Knapsack sprayer fitted with hollow cone nozzles. Water volume for spray was1.5 lit per 30 sq. m (500 lit/ha). Observation on disease severity and incidence were recorded at 40, 50, 60 and 70 days after sowing from ten randomly selected plants. The active ingredient of Equation Pro 42 SC is Famoxadone and Cymoxanil and is a systemic and contact fungicide.

Standard and recommended packages of practices were followed to raise the crop. 


\section{Treatment details for bio-efficacy and phytotoxicity}

\begin{tabular}{|c|l|c|c|c|}
\hline $\begin{array}{c}\text { Sl. } \\
\text { No. }\end{array}$ & \multicolumn{1}{|c|}{ Treatment } & $\begin{array}{c}\text { Dosage } \\
\text { (g a.i. } / \text { /ha }\end{array}$ & $\begin{array}{c}\text { Dose } \\
\text { formulated } \\
\text { product/ha }\end{array}$ & \multicolumn{1}{|c|}{ Time of application } \\
\hline $\mathbf{1 .}$ & $\begin{array}{l}\text { Equation Pro } 42 \\
\text { SC }\end{array}$ & 105 & 250 & $\begin{array}{l}1^{\text {st }} \text { just after disease appearance \& rest at } \\
10 \text { days after } 1^{\text {st }} \text { spray }\end{array}$ \\
\hline $\mathbf{2 .}$ & $\begin{array}{l}\text { Equation Pro } 42 \\
\text { SC }\end{array}$ & 157.5 & 375 & $\begin{array}{l}1^{\text {st }} \text { just after disease appearance \& rest at } \\
10 \text { days after } 1^{\text {st }} \text { spray }\end{array}$ \\
\hline $\mathbf{3 .}$ & $\begin{array}{l}\text { Equation Pro 42 } \\
\text { SC }\end{array}$ & 210 & 500 & $\begin{array}{l}1^{\text {st }} \text { just after disease appearance \& rest at } \\
10 \text { days after } 1^{\text {st }} \text { spray }\end{array}$ \\
\hline $\mathbf{4 .}$ & $\begin{array}{l}\text { Equation Pro } 42 \\
\text { SC }\end{array}$ & 262.5 & 625 & $\begin{array}{l}1^{\text {st }} \text { just after disease appearance \& rest at } \\
10 \text { days after } 1^{\text {st }} \text { spray }\end{array}$ \\
\hline $\mathbf{5 .}$ & $\begin{array}{l}\text { Curzate M8 72\% } \\
\text { WP }\end{array}$ & 1080 & 1500 & $\begin{array}{l}1^{\text {st }} \text { just after disease appearance \& rest at } \\
10 \text { days after } 1^{\text {st }} \text { spray }\end{array}$ \\
\hline *6 & $\begin{array}{l}\text { Equation Pro } 42 \\
\text { SC }\end{array}$ & 840 & 2000 & $\begin{array}{l}1^{\text {st }} \text { just after disease appearance \& rest at } \\
10 \text { days after } 1^{\text {st }} \text { spray }\end{array}$ \\
\hline $\mathbf{7 .}$ & $\begin{array}{l}\text { Ridomyl 72\% } \\
\text { WP }\end{array}$ & 1080 & 1500 & $\begin{array}{l}1^{\text {st }} \text { just after disease appearance \& rest at } \\
10 \text { days after } 1^{\text {st }} \text { spray }\end{array}$ \\
\hline $\mathbf{8 .}$ & Untreated control & $\begin{array}{l}\text { Water } \\
\text { spray }\end{array}$ & Water spray & \\
\hline
\end{tabular}

*Treatment for recording Phytotoxicity observations only.

Method of disease scoring (0-9 rating scale) was done using GWBASIC computer was as described by C.D. Mayee and V.V. software package.

Datar (1986). Analysis of variance (ANOVA)

Disease score ratting scale:

$\begin{array}{cll}\underline{\text { Scale }} & & \text { Description } \\ 0 & & \text { No incidence } \\ 1 & & \text { Less than 1\% area affected } \\ 3 & & \text { 1-5\% Leaf area affected } \\ 5 & & \text { 6-25\% Leaf area affected } \\ 7 & \text { 26-50\% Leaf area affected } \\ 9 & \text { 61-100\% Leaf area affected }\end{array}$

Ten plants were randomly selected from each plot and the leaves were graded on a $0-9$

scale, Percent Disease Index (PDI) was calculated using the following formula:

$$
\text { PDI }=\frac{\text { Sum of all numerical ratings }}{\text { Total plants (leaves) observed } \mathrm{x} \text { Maximum ratings scale }} \times 100
$$


The PDI values and percent disease incidence were transformed by angular transformation and analyzed statistically. The yield data $\mathrm{kg} / \mathrm{plot}$ was also analyzed statistically.

\section{Results and Discussion}

The appearance of the first visible symptom of the disease of Gherkin was recorded at 37 Days after sowing (DAS). The disease incidence and severity of downy mildew disease of gherkin has been presented in Table 1 and 2. It was found that Equation Pro $42 \mathrm{SC}$ and their different dosage significantly reduced the disease over control and recommended dosage. Curzate M8 72 WP \% and Ridomyl $72 \%$ also reduced the diseases in gherkin. Mohamed et al., revealed that
Famoxadone +Cymoxanilare very much effective to control downy mildew disease in cucumber and which is a cucurbit. However, they showed differential effects in controlling the disease under field condition. The first spray was done at $40 \mathrm{DAS}$. The fungicide Equation Pro 42 SC (262.50 g a.i./ha) showed significant minimum disease severity i.e. $11.40 \%$ and maximum disease control i.e $82.41 \%$ over control, followed by $\mathrm{T}_{3}(210 \mathrm{~g}$ a.i./ha) where, disease severity is $14.25 \%$ and disease control is $76.24 \%$ over $\mathrm{T}_{9}$ (control plot). $T_{4}$ and $T_{3}$ are at par. The significant lowest disease incidence and highest control was recorded in $\mathrm{T}_{4}$ (Equation Pro 42 SC@262.5 g a.i/ha) and $T_{3}$ (Equation Pro 42 SC @ $210.0 \mathrm{~g}$ a.i/ha) treated plots, $\mathrm{T}_{4} \& \mathrm{~T}_{3}$ are statistically at par.

Table.1 Effect of equation pro against downy mildew disease of Gherkin

\begin{tabular}{|c|c|c|c|c|c|c|c|}
\hline \multirow[t]{3}{*}{ Treatment } & \multirow[t]{3}{*}{ gma.i./ha } & \multicolumn{4}{|c|}{ Percentage of incidence } & \multirow{3}{*}{$\begin{array}{c}\text { Disease } \\
\text { control } \\
\%\end{array}$} & \multirow{3}{*}{$\begin{array}{l}\text { Fruit } \\
\text { Yield } \\
\text { kg/ha }\end{array}$} \\
\hline & & \multicolumn{4}{|c|}{ Days after sowing } & & \\
\hline & & 40 & 50 & 60 & 70 & & \\
\hline $\begin{array}{l}\text { T}_{1} \text {-Equation Pro } 42 \\
\text { SC }\end{array}$ & 105 & $\begin{array}{c}0.0 \\
(0.72)\end{array}$ & $\begin{array}{l}13.26 \\
(3.62)\end{array}$ & $\begin{array}{l}27.81 \\
(5.22)\end{array}$ & $\begin{array}{l}42.98 \\
(6.61)\end{array}$ & 32.80 & 706.67 \\
\hline $\begin{array}{l}\mathrm{T}_{2} \text {-Equation Pro } 42 \\
\text { SC }\end{array}$ & 157.5 & $\begin{array}{c}2.99 \\
(1.67)\end{array}$ & $\begin{array}{l}15.95 \\
(4.01)\end{array}$ & $\begin{array}{l}28.84 \\
(5.40)\end{array}$ & $\begin{array}{l}39.17 \\
(6.31)\end{array}$ & 38.75 & 786.67 \\
\hline $\begin{array}{l}T_{3}-\text { Equation Pro } \\
42 \mathrm{SC}\end{array}$ & 210.0 & $\begin{array}{c}2.12 \\
(1.55)\end{array}$ & $\begin{array}{c}6.66 \\
(2.67)\end{array}$ & $\begin{array}{c}9.95 \\
(3.26)\end{array}$ & $\begin{array}{l}13.33 \\
(3.73)\end{array}$ & 79.15 & 1560.00 \\
\hline $\begin{array}{l}\mathrm{T}_{4} \text {-Equation Pro } \\
42 \mathrm{SC}\end{array}$ & 262.5 & $\begin{array}{c}1.06 \\
(1.13)\end{array}$ & $\begin{array}{c}6.66 \\
(2.67)\end{array}$ & $\begin{array}{c}7.98 \\
(3.18)\end{array}$ & $\begin{array}{r}13.33 \\
(3.73)\end{array}$ & 79.15 & 1573.33 \\
\hline $\begin{array}{l}\mathrm{T}_{5} \text {-Curzate M8 } 72 \\
\text { WP \% }\end{array}$ & 1080 & $\begin{array}{c}3.64 \\
(1.86)\end{array}$ & $\begin{array}{c}9.95 \\
(3.26)\end{array}$ & $\begin{array}{l}12.40 \\
(3.62)\end{array}$ & $\begin{array}{l}16.62 \\
(4.16)\end{array}$ & 74.01 & 1053.33 \\
\hline $\mathbf{T}_{6}$-Ridomyl 72\% & 1080 & $\begin{array}{c}1.06 \\
(1.13)\end{array}$ & $\begin{array}{c}7.82 \\
(3.39)\end{array}$ & $\begin{array}{l}17.67 \\
(4.29)\end{array}$ & $\begin{array}{l}18.71 \\
(4.41)\end{array}$ & 70.74 & 1000.00 \\
\hline $\mathbf{T}_{7}$-Control & $\begin{array}{l}\text { Water } \\
\text { spray }\end{array}$ & $\begin{array}{c}1.06 \\
(1.13)\end{array}$ & $\begin{array}{l}35.33 \\
(5.93)\end{array}$ & $\begin{array}{l}60.59 \\
(7.82)\end{array}$ & $\begin{array}{l}63.96 \\
(8.02)\end{array}$ & -- & 333.33 \\
\hline S.Em \pm & & 0.49 & 0.30 & 0.37 & 0.2 & - & 68.00 \\
\hline CD at $5 \%$ & & NS & 0.92 & 1.13 & 0.61 & - & 208.00 \\
\hline
\end{tabular}

Figures in the parenthesis indicate angular transformed values 
Table.2 Effect of equation pro against downy mildew disease of Gherkin

\begin{tabular}{|c|c|c|c|c|c|c|}
\hline \multirow[t]{3}{*}{ Treatment } & \multirow[t]{3}{*}{ gma.i./ha } & \multicolumn{4}{|c|}{ Percentage of severity } & \multirow[t]{3}{*}{$\begin{array}{c}\text { Disease } \\
\text { control \% }\end{array}$} \\
\hline & & \multicolumn{4}{|c|}{ Days after sowing } & \\
\hline & & 40 & 50 & 60 & 70 & \\
\hline $\begin{array}{l}\text { T }_{1} \text {-Equation Pro } 42 \\
\text { SC }\end{array}$ & 105 & $\begin{array}{c}0.0 \\
(0.71)\end{array}$ & $\begin{array}{l}27.43 \\
(4.93)\end{array}$ & $\begin{array}{l}34.57 \\
(5.78)\end{array}$ & $\begin{array}{l}43.49 \\
(6.65)\end{array}$ & 27.50 \\
\hline $\begin{array}{l}\text { T }_{2} \text {-Equation Pro } 42 \\
\text { SC }\end{array}$ & 157.5 & $\begin{array}{c}3.69 \\
(1.88)\end{array}$ & $\begin{array}{l}22.93 \\
(4.85)\end{array}$ & $\begin{array}{l}29.38 \\
(5.48)\end{array}$ & $\begin{array}{l}41.83 \\
(6.52)\end{array}$ & 30.31 \\
\hline $\begin{array}{l}\text { T }_{3} \text {-Equation Pro } 42 \\
\text { SC }\end{array}$ & 210.0 & $\begin{array}{c}3.69 \\
(1.88)\end{array}$ & $\begin{array}{c}6.90 \\
(2.72)\end{array}$ & $\begin{array}{l}10.42 \\
(3.31)\end{array}$ & $\begin{array}{l}14.25 \\
(3.87)\end{array}$ & 76.24 \\
\hline $\begin{array}{l}\text { T }_{4} \text {-Equation Pro } 42 \\
\text { SC }\end{array}$ & 262.5 & $\begin{array}{c}1.96 \\
(1.33)\end{array}$ & $\begin{array}{r}7.87 \\
(2.89)\end{array}$ & $\begin{array}{l}10.17 \\
(3.44)\end{array}$ & $\begin{array}{l}11.40 \\
(3.61)\end{array}$ & 82.41 \\
\hline $\begin{array}{l}\text { T }_{5} \text {-Curzate M8 } 72 \\
\text { WP \% }\end{array}$ & 1080 & $\begin{array}{c}3.69 \\
(1.88)\end{array}$ & $\begin{array}{l}15.30 \\
(4.28)\end{array}$ & $\begin{array}{c}18.1 \\
(4.89)\end{array}$ & $\begin{array}{l}18.79 \\
(4.69)\end{array}$ & 68.67 \\
\hline $\mathbf{T}_{6}$-Ridomyl 72\% & 1080 & $\begin{array}{c}1.23 \\
(1.17)\end{array}$ & $\begin{array}{l}17.04 \\
(4.48)\end{array}$ & $\begin{array}{l}18.28 \\
(4.58) \\
\end{array}$ & $\begin{array}{l}21.09 \\
(4.66)\end{array}$ & 64.84 \\
\hline $\mathbf{T}_{7}$-Control & $\begin{array}{l}\text { Water } \\
\text { spray }\end{array}$ & $\begin{array}{c}1.23 \\
(1.17)\end{array}$ & $\begin{array}{l}43.77 \\
(6.55)\end{array}$ & $\begin{array}{l}58.60 \\
(7.70)\end{array}$ & $\begin{array}{l}59.99 \\
(7.78)\end{array}$ & - \\
\hline S.Em \pm & & 0.54 & 0.28 & 0.20 & 0.08 & - \\
\hline CD at $5 \%$ & & 1.65 & 0.85 & 0.61 & 0.24 & - \\
\hline
\end{tabular}

Figures in the parenthesis indicate angular transformed values

Table.3 Phytotoxicity effect of equation pro against downy mildew disease of Gherkin

\begin{tabular}{|c|c|c|c|c|c|c|c|}
\hline \multirow{2}{*}{ Treatment } & \multicolumn{7}{|c|}{$\mathbf{3 , 5 , 7}$ and 10 days after each application } \\
\cline { 2 - 8 } & $\begin{array}{c}\text { Grain } \\
\text { discolouration }\end{array}$ & Blackening & Burning & Malformation & Stunting & Necrosis & Chlorosis \\
\hline $\mathbf{T}_{\mathbf{1}}$ & 0 & 0 & 0 & 0 & 0 & 0 & 0 \\
\hline $\mathbf{T}_{\mathbf{2}}$ & 0 & 0 & 0 & 0 & 0 & 0 & 0 \\
\hline $\mathbf{T}_{\mathbf{3}}$ & 0 & 0 & 0 & 0 & 0 & 0 & 0 \\
\hline $\mathbf{T}_{\mathbf{4}}$ & 0 & 0 & 0 & 0 & 0 & 0 & 0 \\
\hline $\mathbf{T}_{\mathbf{5}}$ & 0 & 0 & 0 & 0 & 0 & 0 & 0 \\
\hline $\mathbf{T}_{\mathbf{6}} *$ & 0 & 0 & 0 & 0 & 0 & 0 & 0 \\
\hline $\mathbf{T}_{\mathbf{7}} *$ & 0 & 0 & 0 & 0 & 0 & 0 & 0 \\
\hline
\end{tabular}

*Treatment for recording phytotoxicity observations only.

After 70 days of sowing 13.33 percent disease incident was observed in $\mathrm{T}_{4}$ treated plants and it is same in $\mathrm{T}_{3}$. Lowest disease incident may be due to death of sporangia or zoospore (Jin et al., 2009). Curzate M8 72 and Ridomyl $72 \%$ also showed significant disease control percentage i.e $74.01 \%$ and $70.74 \%$, respectively. Bhat et al., (2018) coined that Ridomyl and Curzate showed significant minimum disease severity. Ridomyl is very much useful against downy mildew of cucumber, a cucurbit (Anthony et al., 2012; 
Hang-cheng et al., 2009; Robak et al., 1995; Sharma et al., 2003 and Samoucha and Cohen, 1998). But, the significant higher yield was found when Equation Pro 42 SC (262.50 g a.i. /ha) sprayed over crop and is at par with $\mathrm{T}_{3}$ treated plots. Mohamed et al., also found satisfactory fruit yield when it is treated with Famoxadone + Cymoxanil against downy mildew disease.

Equation Pro 42 SC showed no phytotoxic symptom sprayed to the gherkin crop up to 4 numbers of spraying at their different dosages mentioned in the report (Table 3). No fruit discolouration, blackening, burning and malformation were found at 10 days after last application. No phytotoxic effect like stunting, necrosis, and chlorosis was noticed at 3, 5, 7 and 10 days after each spraying. It controls diseases completely and its antisporulent and spore killing activity also checks further disease spread.

Finding and conclusion of the study are as follows:

The severity of the downy mildew disease of gherkin was the lowest when Equation Pro 42 SC was sprayed over the crop @ $262.50 \mathrm{~g}$ a.i./ha, it was at par with Equation Pro 42 SC $210.0 \mathrm{~g}$ a.i/ha. Percentage of disease control was $82.41 \& 76.24$ respectively. It may be concluded that higher yield and significant disease control was recorded when Equation Pro 42 SC was sprayed over the crop @ 262.50g a.i/h. and it was at par when Equation Pro 42 SC $210.0 \mathrm{~g}$ a.i/ha was sprayed over the crop.

\section{References}

Anthony, P.K., and Virginia, B.D. 2012. Controlling powdery mildew on cucurbit rootstock seedlings in the greenhouse with fungicides and biofungicides. Crop protection.42: 338-
44.

Bernhaedt, E., Dodson, J. and Waterson, J. 1988. Cucurbit Diseases: a Practical Guide for Seeds Men, Growers and Agricultural Advisors. Saticoy, Petoseed Co.

Bhat, J. A., Rashid, R., Dar, W. A. and Bhat, R. A. 2018. Efficacy of different fungicide for the management of downy mildew of cucumber grown under low plastic tunnel. Int. J. Pure App. Biosci., 6(2): 884-890.

Han-cheng, W., Ming-guo, Z., Jian-xin, W., Chang-jung, C., Hong-xia, L. and Haiyan, S. 2009. Biological mode of action of dimethomorph on

Pseudoperonospora cubensis and its systemic activity in cumber. Agri. Sci. in China. 8: 172-18.

Hansen, M. A. 2000. Downy Mildew of Cucurbits. Publication Number 450707. Virginia Cooperative Extension. Available http://www.ext.vt.edu/pubs/plantdisease fs/ 450- 707/450-707.html

Hausbeck, M.K., Morrice, J. and Linderman, S. 2014. Monitoring and managing cucurbit downy mildew. Michigan State University Extension.

Jin, T., Sun, D., Su, J.Y., Zhang, H. and Sue, H. 2009. Antimicrobial efficacy of zinc oxide quantum dots against L. monocytogenes, S. enteritidis, and E. coli O157:H7. Journal of Food Science. 74: M46-M52.

Keinath, P. A. 2014. Cucurbit downy mildew management. Clemson University Cooperative Extension. IL-90, Rev.

Mayee, C. D and Datar, V.V. 1986. Phytopathometry; Pub: Marathawada Agric. University, Parbhani, India. Pp. 214.

Mohamed, A., Hamza, A. and Derbalah, A. 2016. Recent approaches for controlling downy mildew of cucumber under greenhouse conditions. Plant Protect. 
Sci. 52: 1-9.

Palti, J. and Cohen, Y.1980. Downy mildew of Cucurbits (Pseudoperonospora cubensis): the Fungus and its hosts, distribution, epidemiology and control. Phytoparasitica. 8(2): 109-147.

Robak, J. 1995. Epidemiology and control of cucumber downy mildew (Pseudoperonospora cubensis). Warzywniczy 43: 5-18.

Rodrigo, M. and Alvarruiz, A. 1998. "The influence of fermentation and pasteurization on the texture of cucumber pickles". Journal of food engineering. 7(2): 113-125.

Samoucha, Y. and Cohen, Y. 1998. Synergism in fungicide mixtures against Pseudoperonospora cubensis. Phytoparasitica. 16: 337-42.

Sharma, D.R., Gupta, S.K. and Shyam, K,R. 2003. Studies on downy mildew of cucumber caused by Pseudoperonospora cubensis and its management. Indian J. Mycol. Plant Pathol. 33: 246-51.

\section{How to cite this article:}

Sanjay Bairagi, P.S. Nath, M.K. Pandit and Arindam Das. 2019. Gherkin Downy Mildew Disease and Its Response to Different Fungicides. Int.J.Curr.Microbiol.App.Sci. 8(03): 844850. doi: https://doi.org/10.20546/ijcmas.2019.803.101 